\title{
Inclusion Body Myositis
}

\author{
Mazen M. Dimachkie, M.D. ${ }^{1}$ and Richard J. Barohn, M.D. ${ }^{1}$ \\ ${ }^{1}$ Department of Neurology, The University of Kansas Medical Center, Kansas City, Kansas
}

\begin{abstract}
The idiopathic inflammatory myopathies are a group of rare disorders that share many similarities. These include dermatomyositis (DM), polymyositis (PM), necrotizing myopathy (NM), and sporadic inclusion body myositis (IBM). Inclusion body myositis is the most common idiopathic inflammatory myopathy after age 50 and it presents with chronic proximal leg and distal arm asymmetric mucle weakness. Despite similarities with PM, it is likely that IBM is primarily a degenerative disorder rather than an inflammatory muscle disease. Inclusion body myositis is associated with a modest degree of creatine kinase (CK) elevation and an abnormal electromyogram demonstrating an irritative myopathy with some chronicity. The muscle histopathology demonstrates inflammatory exudates surrounding and invading nonnecrotic muscle fibers often times accompanied by rimmed vacuoles. In this chapter, we review sporadic IBM. We also examine past, essentially negative, clinical trials in IBM and review ongoing clinical trials. For further details on DM, PM, and NM, the reader is referred to the idiopathic inflammatory myopathies chapter.
\end{abstract}

\section{Keywords}

inclusion body myositis; idiopathic inflammatory myopathies; polymyositis; pathophysiology; therapy; prognosis

\section{Epidemiology}

Inclusion body myositis is a sporadic disorder with a male-to-female ratio of 3:1. Interestingly, the age-adjusted prevalence of IBM in people over the age of 50 is $3.5 / 100,000$, making it the most common idiopathic inflammatory myopathy in this age group. ${ }^{1}$ The prevalence of IBM in the Netherlands is 4.9 cases per million inhabitants ${ }^{2}$ and overall 9.3 per million Australian inhabitants. ${ }^{1}$ In an Olmsted county population study, the estimated incidence of IBM, adjusted for sex and age to the 2000 U.S. Census population, was 7.9 cases per million inhabitants with a prevalence of 70 cases per million inhabitants. ${ }^{3}$ In the Mayo Clinic series of 107 patients with pathologic features of PM or IBM, 64 had IBM pathology, and 16 of 43 cases with PM pathology demonstrated IBM clinical features. ${ }^{4}$ The later clinical phenotype is predictive of the diagnosis and of poor treatment response. IBM is rare in African Americans and in non-Caucasians.

Inclusion body myositis is the most common myopathy after age 50, but should be considered in patients with appropriate symptoms who are older than 30 . Symptom onset before age 60 occurs in 18 to $20 \%$ of patients, ${ }^{5,6}$ with a frequent delay in diagnosis of 5 to 8 years from IBM symptom onset. $2,5,7,8$ 


\section{Clinical Presentation}

The clinical presentation of IBM is quite distinct from that of PM, DM, or NM (Table 1). The classic IBM pattern occurs in the majority of cases and consists of coexistent proximal leg and distal arm weakness. ${ }^{9,10}$ Most commonly IBM manifests early on as slowly progressive quadriceps muscle weakness leading to falls or difficulty standing up. ${ }^{11}$ Less common initial complaints include finger flexor weakness and atrophy, foot drop, or dysphagia. Rare presentations include sparing of the quadriceps muscles with prominent forearm muscle weakness. Up to $82 \%$ of cases have marked asymmetry especially notable in the non-dominant hand deep (distal) finger flexor muscles. Due to the several year delay in presentation, asymmetric atrophy and weakness of wrist and finger flexors (see Fig. 1A) and quadriceps muscles (see Fig. 1B) are evident in most cases on presentation, and lead to disability from loss of dexterity and early falls. This pattern should prompt consideration of inclusion body myositis in the elderly patient. Sparing of the thenar and hypothenar muscles helps distinguish IBM from a myotomal disease like amyotrophic lateral sclerosis. Wrist and finger flexors are weaker in IBM than the corresponding extensors and the shoulder abductors. This is in contrast to the proximal predominant pattern of weakness seen in DM, PM, and NM. Similarly, knee extension weakness out of proportion to hip flexion weakness is supportive of IBM. Granulomatous myositis may mimic the inclusion body myositis weakness pattern and may be steroid-responsive. ${ }^{12}$

Involvement of the tibialis anterior muscle occurs in $10 \%$ of IBM patients leading to ankle dorsiflexion weakness. Dysphagia affects up to $70 \%$ of patients and can be a significant problem. ${ }^{5,13}$ Mild to moderate facial weakness is frequently demonstrated. Although mostly asymptomatic, $30 \%$ of patients may have clinical and/or electrophysio-logic evidence of a sensory neuropathy. Patellar reflexes may be lost due to severe quadriceps weakness and atrophy. Progression of leg weakness results in falls ultimately leading to wheelchair confinement in 10 to 15 years from onset.

Table 2 lists the Griggs diagnostic criteria for IBM. Table 3 includes the 2010 revised criteria. ${ }^{14}$ The purpose of modifying the criteria was to facilitate the diagnosis of patients who fulfill clinical criteria for IBM, but do not have the pathologic features set forth by the Griggs criteria. ${ }^{15}$ Besides the Griggs IBM criteria of pathologically defined IBM, they added two new categories of IBM diagnosis for suspected patients presenting with weakness onset after 35 years of age and lasting at least for 12 months. These are clinically defined IBM and possible IBM. In clinically defined IBM, weakness is more marked in finger flexors than shoulder abductors and it is more pronounced in knee extensors as compared with hip flexor muscles. The pathologic features of clinically defined IBM include invasion of nonnecrotic fibers by mononuclear cells or rimmed vacuoles or increased MHC-1 expression on the surface of muscle fibers. Hence, rimmed vacuoles are not an essential histopathologic finding for this category and the same is true of intracellular amyloid deposits and the15- to 18-nm tubulofilamentous inclusions. The criteria for possible IBM are nearly identical to those of clinically defined IBM with one exception relating to the pattern of weakness. Namely, weakness must be more marked in finger flexors than shoulder abductors or it should be more pronounced in knee extensors as compared with hip flexor muscles. The pathologic criteria are the same as those of clinically defined IBM.

\section{Associated Conditions}

Though IBM is felt to be a neurodegenerative disorder, there is some association with autoimmune disorders. Systemic lupus erythematosus, Sjögren's syndrome, thrombocytopenia, and sarcoidosis have been reported in up to $15 \%$ with IBM. There is no increased risk of myocarditis, interstitial lung disease, or malignancy in IBM. ${ }^{16}$ 


\section{Laboratory Testing}

Serum CK level may be normal or elevated up to 10 times the upper normal limit. On occasion, it may be as high as 20 times the normal limit. ANA is positive in $20 \%$ of IBM patients. IBM patients have an increased prevalence of the HLA DR $30301 / 0302$ phenotype. ${ }^{17}$

\section{Electrophysiology}

Nerve conduction studies reveal a mild sensory axonal peripheral polyneuropathy in up to $30 \%$ of patients with IBM. Otherwise, the electrodiagnostic findings are similar to those in DM, PM, and NM with evidence of an irritative myopathy (e. g., fibrillation potentials and positive sharp waves). However, in one out of three IBM cases, the motor unit potentials are mixed myopathic and neuropathic. These "pseudo" neurogenic changes are due to reinnervation of denervated and split muscle fibers. Large amplitude polyphasic MUAPs may also be seen in chronic PM and DM. In some cases, the neurogenic motor unit action potentials in IBM may be sufficiently dense to overshadow the myopathic changes, leading to a misdiagnosis of motor neuron disease. In these cases, the clinical history, pattern of weakness, prolonged survival, and lack of associated upper motor neuron findings suggest the correct diagnosis and prompt the need for a confirmatory muscle biopsy.

\section{Muscle Imaging}

Imaging of leg muscles demonstrates preferential involvement of the quadriceps and gastrocnemius medialis followed by the soleus muscle. ${ }^{18}$ Fifty percent of IBM patients have a distal and asymmetric involvement. ${ }^{18}$ Hyperintensity on STIR sequence is associated with prominent fatty atrophy in $80 \%$ of cases. In another study, muscles most frequently infiltrated with fat were the long finger flexors, anterior thigh muscles (relatively sparing the rectus femoris), and all muscles of the lower leg, preferentially affecting the medial gastrocnemius muscle. ${ }^{19}$ The amount of fatty infiltration correlated well with disease severity, disease duration and CK levels. A study of positron emission tomography using Pittsburgh Compound B (PIB), an in vivo marker of amyloid $\beta$ in the brains of patients with Alzheimer's disease, detected increased uptake levels in the gastrocnemius muscle of IBM patients. ${ }^{20}$

\section{Muscle Histopathology}

Inclusion body myositis was originally believed to be a primary inflammatorymyopathy. However, recent pathologic evidence supports a neurodegenerative etiology. In addition to endomysial inflammation (Fig. 2A), the presence of small groups of atrophic fibers, eosinophilic cytoplasmic inclusions and most notably multiple myofibers with one or more rimmed vacuoles lined with granular material is highly supportive of a pathologic diagnosis of IBM(seeFigs. 2B, 2C).However, these vacuoles may only be detectable on second or third muscle biopsies performed on treatment-refractory patients manifesting the phenotype of IBM and histopathologic findings of PM. ${ }^{9}$ Furthermore, eosinophilic cytoplasmic inclusions are rarely seen in IBM. These can be better visualized by an immunostain directed against phosphorylated tau (SMI-31).

Patients who have typical IBM clinical features, but few inflammatory cells or few rimmed vacuoles can be difficult to diagnose pathologically. ${ }^{9}$ Furthermore, patients who have steroid-responsive PM may have a few rimmed vacuoles. ${ }^{21}$ Some IBM patients are mislabeled as PM when no vacuoles are found even though they have the classic clinical phenotype. ${ }^{4}$ These patients may require a second or even third muscle biopsy to find the typical IBM histopathologic features. Although typical histologic features are necessary for 
a pathologically confirmed diagnosis, revised diagnostic criteria allow for clinically defined IBM (Table 3).

The Congo red stain demonstrates congophilic material in vacuolated fibers that is likely to represent amyloid deposition. This small amount of amyloid-positive material is present within or next to the vacuoles. Fluorescent methods for detecting amyloid material are even more sensitive than Congo red staining. There are an increased number of ragged red fibers and COX negative fibers. Some nuclei containing eosinophilic inclusions appear to be enlarged within the vacuoles. There is an increased likelihood of finding 15- to18-nanometer cytoplasmic and intranuclear tubulofilamentous inclusions on electron microscopy when at least three vacuolated fibers are examined. The presence of more than one rimmed vacuole, more than one group of atrophic fibers per high-power field, and of endomysial inflammation is said to be $95 \%$ predictive of finding the filamentous inclusions by electron microscopic examination. ${ }^{5}$ The eosinophilic cytoplasmic inclusions correspond to the tubulofilamentous inclusions seen on electron microscopy.

There are several histopathologic similarities between PM and IBM. ${ }^{22}$ In both, intact myofibers are surrounded and invaded by endomysial inflammatory cells that consist of macrophages and cytotoxic CD8+ T cells with MHC-1 expression on the surface of necrotic and nonnecrotic myofibers. In addition, myeloid dendritic cells surround nonnecrotic fibers and present antigen to the CD8+ lymphocytes. However, mononuclear cells invade nonnecrotic muscle fibers more frequently in IBM than in PM.

\section{Pathogenesis}

The pathogenesis of IBM remains unknown. Proposed modes of injury in IBM include autoimmune (cytotoxic T cells, myeloid dendritic cells, B cells, and a newly discovered IBM autoantibody) and degenerative pathways. ${ }^{23}$ As in PM, clonally restricted cytotoxic Tcells invade and destroy nonnecrotic muscle fibers through perforin, granzyme A, and granulysin pathways. The frequency of muscle fiber invasion in IBM is higher than that observed for vacuolated fibers or fibers with amyloid deposits. In IBM muscle, myeloid dendritic cells (mDCs) are believed to serve as antigen-presenting cells. ${ }^{22}$ These mDCs help the maturation of naïve CD8+ T cells into cytotoxic T cells and surround and invade nonnecrotic muscle fibers. Microarray studies showed an abundance of immunoglobulin transcripts in IBM muscle ${ }^{24}$ and led to the recognition of plasma cells that are antigen directed and clonally expanded. ${ }^{25}$ There is a modest upregulation of type 1 interferon (IFN1) genes in IBM muscle. However, unlike PM, IFN1 is not modestly upregulated in blood derived from IBM cases. A circulating autoantibody to an $\sim 43 \mathrm{kDa}$ human muscle protein is present in $52 \%$ of the IBM samples, ${ }^{26}$ but the identity and significance of this protein remain unclear.

However, other lines of evidence point toward a degenerative pathophysiology. IBM does not respond to immunomodulatory therapy, a fact that strongly argues against IBM being a primarily immune-mediated disease. The degenerative pathogenesis model of IBM stems from the immunohistochemical identification in vacuolated muscle fibers of protein aggregates (amyloid $\beta$, hyperphosphorylated tau, ubiquitin, neurofilament heavy chain, presenilin, and parkin, among others) often associated with other neurodegenerative diseases. These aggregates are postulated to occur due to aberrant protein misfolding and accumulation. ${ }^{27}$ Potential contributory mechanistic pathways include the $26 \mathrm{~S}$ proteasome system, various heat shock proteins, and impaired autophagy. ${ }^{28}$ There is also overexpression of $\beta$ crystalline, a heat shock chaperone protein, that might be an upstream step in the pathogenesis of IBM. However, proponents of the autoimmune theory of IBM note the lack of critically supported data that demonstrate the presence of those proteins on Western blot 
of IBM muscle specimens and refer to the cross reactivity of many $\beta$ amyloid antibodies with the $\beta$ amyloid precursor protein. ${ }^{29}$ In addition, $\beta$ amyloid precursor protein is secreted by inflammatory cells, and has therefore been detected in PM and DM tissues in addition to IBM. Subsequently, Askanas' group reported that IBM muscle samples had accumulation of toxic amyloid $\beta$ oligomers on dot immunoblots with a variety of molecular weights and intensity, but none of the control muscle biopsies had amyloid $\beta$ oligomers. ${ }^{30}$ Of interest, a recent positron emission tomography study using PIB, a marker of $\beta$ amyloid, detected increased uptake levels in gastrocnemius muscles of IBM patients. ${ }^{20}$

There is also myonuclear degeneration early on in IBM because the majority of rimmed vacuoles are lined with nuclear membrane proteins. IBM myonuclei are often abnormally filled with neurofilaments and this may be the earliest detectable pathologic change in IBM. $^{31}$

In IBM, myofibers contain nonnuclear sarcoplasmic Tar DNA binding protein 43 (TDP-43) accumulations together with a reduction of the normal nuclear TDP-43 content. This suggests that TDP-43 has redistributed from nuclei to sarcoplasm in a large percentage of IBM myofibers. ${ }^{32}$ The extra-nuclear accumulation of TDP-43 is toxic to cells through its binding to RNA. Therefore, IBM muscle accumulates multiple toxic protein aggregates suggesting a disorder of protein homeostasis.

\section{Therapy}

IBM has been refractory to all treatments known to be effective in DM, PM, and NM. In general, IBM patients are refractory to prednisone, ${ }^{5}$ except for the occasional patient who experiences a transient and mild improvement in response to corticosteroids early on in their disease course. ${ }^{7}$ This initial response to corticosteroid therapy may be dramatic in some and followed by progressive resistance to therapy over 3 to 6 years. ${ }^{33}$ Therefore, treatment with corticosteroids may be considered for the rare case that lacks the typical IBM pattern of weakness early on in the course of the disease. Otherwise, corticosteroid treatment is not recommended for typical IBM cases. In a long-term observational study of 136 patients, those who received immunosuppressive treatments (52\%) were at last assessment more severely affected on disability scales and on the sporadic inclusion body myositis weakness composite index. ${ }^{34}$ The first stage of disease progression toward handicap for walking was more rapid among patients receiving immunosuppressive treatments. Therefore, immunosuppressive treatments do not ameliorate IBM's natural course. Most intravenous immunoglobulin (IVIg) studies did not demonstrate clinically meaningful efficacy ${ }^{35-37}$ despite an earlier encouraging report. ${ }^{5}$ Subsequent randomized controlled trials of IVIg without corticosteroids ${ }^{36}$ — and with corticosteroids ${ }^{37}$ — did not show any benefit.

Two Muscle Study Group randomized studies did not show any efficacy of $\beta$-interferon-1a at standard ${ }^{38}$ and high doses. ${ }^{39}$ Methotrexate in a prolonged randomized controlled trial was also not effective..$^{40}$ Despite promising results of antithymocyte globulin in a pilot trial, ${ }^{41}$ there is no ongoing controlled trial in IBM. A small pilot randomized trial of oxandrolone, an androgen receptor agonist, demonstrated a borderline significant effect in improving whole-body strength and amore significant benefit in the upper extremities maximal voluntary isometric contraction (MVICT). ${ }^{42}$ In a small pilot trial of etanercept, there was no clinically meaningful improvement in handgrip at 12 months, and no further clinical trials of TNF blockers are planned. ${ }^{43}$ Alemtuzumab, which was recently tested in an open-label small proof-of-principle study, showed a reduction in muscle CD3 lymphocytes, but it did not significantly improve IBM patients' strength or function. ${ }^{44}$ More recently, an open-label pilot trial of oral simvastatin $40 \mathrm{mg}$ daily for 12 months was conducted to evaluate its safety and tolerability IBM patients. ${ }^{45}$ Of 14 patients, 10 completed the trial and the treatment 
appeared safe and well tolerated. However, none of the patients showed a significant clinical improvement.

Several novel therapies are being evaluated. A trial of lithium chloride was completed in hope of inducing autophagy, thereby improving clearance of misfolded proteins. The results have not yet been published. Several agents are being evaluated in ongoing clinical trials including Arimoclomol, BYM338, and follistatin gene transfer therapy. Given the putative role of heat shock protein abnormalities in the pathogenesis of IBM, we conducted a twocenter trial of Arimoclomol, a heat shock protein 70 inducer, for the treatment of IBM (see clinicaltrials.gov). This study is currently closed to enrollment and follow-up of the last subject should be completed soon. The other two studies are attempts to increase muscle size and strength or function using different approaches and are listed on clinicaltrials.gov.

\section{Prognosis}

IBM relentlessly progresses to disability without increased mortality. In a 12-year follow-up study, the mean decline in strength was 3.5 and $5.4 \%$ per year as measured by manual muscle testing and quantitative muscle testing, respectively. This resulted in progressive impairment in activities of daily living. ${ }^{46}$ At a mean disease duration of 20 years, all patients were found to be using a wheelchair, seven of them (47\%) being completely wheelchairbound. At 5 years, 10 of 14 cases required a cane or support; at 10 years, most patients (three-fifths) were wheelchair confined. ${ }^{47}$

\section{Exercise}

There certainly is a role for physical therapy, orthotic devices, occupational therapy, a healthy well-balanced diet, and exercise in IBM. A tailored 12-week home-exercise program, 5 days a week for 12 weeks in combination with stationary biking or walks, was found to be safe in 7 patients. ${ }^{48}$ There was no strength deterioration, no change in serum $\mathrm{CK}$, and no increase in muscle inflammation on biopsy. However, the study was not able to show improved muscle function in isokinetic knee extension or flexion assessed by dynamometry, manual muscle test, or by using the Functional Index to assess repetitive muscle function.

Other investigators recently reported the benefits of a 16-week home-exercise program performed twice per day in seven IBM patients, two of whom used a cane and another two a motorized scooter. ${ }^{49}$ The exercises consisted of whole-body sit-to-stand exercises, biceps curl, shoulder press, heel lifts, isometric vastus medialis exercises, and ankle dorsal flexion. Surprisingly, the group improved in all muscle groups, including hip flexion, elbow extension, knee flexion, knee extension, and grip strength. Timed functional tests (to climb one flight of stairs and to walk $30 \mathrm{~m}$ ) were also improved. In another report, the same group of investigators described the effects of an aerobic exercise program using a stationary cycle ergometer at $80 \%$ of the initial maximum heart rate (for 2 minutes less than the total time achieved during maximal aerobic test) combined with the above mentioned resistance isometric and isotonic exercises of the upper and lower limbs in a group of seven IBM cases. ${ }^{50}$ Besides demonstrating safety, they found this exercise routine to improve aerobic capacity and muscle strength in shoulder abduction, hip flexion, hip abduction, and knee flexion, but no changes were noted in knee extension and grip strength. This exercise program was not followed by significant changes in stair time or $30 \mathrm{~m}$ walk test.

Moderate- to low-intensity strength training in combination with vascular occlusion has been proposed as an alternative to heavy resistance-exercise training, especially when high forces' actions are either contraindicated or not achievable. ${ }^{51} \mathrm{~A}$ comparable increase in muscle strength and size in response to this approach has been demonstrated in the frail 
elderly. ${ }^{52}$ In a preliminary case report, a protocol of moderate-intensity resistance training program combined with thigh vascular occlusion at $50 \%$ of total occlusion pressure, done twice per week for 12 weeks was well tolerated with unchanged inflammatory biomarkers. ${ }^{53}$ The patient did not report excessive exertion or pain and there were no signs of worsening inflammation on serial muscle biopsies. This patient improved $\sim 16 \%$ in leg press onerepetition maximum, in the functional timed-up-and-go test (from 16 to 10 seconds), and the thigh cross-sectional area of the quadriceps improved by $4.7 \%$.

These findings indicate that mild to moderate intensity nonfatiguing exercise is safe in IBM. There is a suggestion that exercise might lead to modestly improved muscle strength in some patients. There is conflicting data on the functional benefit of exercise. Large multicenter controlled trials have yet to be conducted to confirm these preliminary findings and to clarify any potential gains from exercise in people with IBM.

\section{Summary}

Inclusion body myositis is the most common idiopathic inflammatory myopathy after age 50. Despite similarities with PM, it is likely that IBM is primarily a degenerative disorder rather than inflammatory muscle disease. The clinical phenotype of IBM is distinctive, presenting with proximal leg and distal arm weakness. IBM is refractory to known pharmacologic treatments. Several novel approaches are currently being studied and there is encouraging data supporting a role for moderate intensity, nonfatiguing exercise in the management of these patients.

\section{References}

1. Phillips BA, Zilko PJ, Mastaglia FL. Prevalence of sporadic inclusion body myositis in Western Australia. Muscle Nerve. 2000; 23(6):970-972. [PubMed: 10842277]

2. Badrising UA, Maat-Schieman M, van Duinen SG, et al. Epidemiology of inclusion body myositis in the Netherlands: a nationwide study. Neurology. 2000; 55(9):1385-1387. [PubMed: 11087787]

3. Wilson FC, Ytterberg SR, St Sauver JL, Reed AM. Epidemiology of sporadic inclusion body myositis and polymyositis in Olmsted County, Minnesota. J Rheumatol. 2008; 35(3):445-447. [PubMed: 18203321]

4. Chahin N, Engel AG. Correlation of muscle biopsy, clinical course, and outcome in PM and sporadic IBM. Neurology. 2008; 70(6):418-424. [PubMed: 17881720]

5. Lotz BP, Engel AG, Nishino H, Stevens JC, Litchy WJ. Inclusion body myositis. Observations in 40 patients. Brain. 1989; 112(Pt 3):727-747. [PubMed: 2543478]

6. Badrising UA, Maat-Schieman ML, van Houwelingen JC, et al. Inclusion body myositis. Clinical features and clinical course of the disease in 64 patients. J Neurol. 2005; 252(12):1448-1454. [PubMed: 15942703]

7. Lindberg C, Persson LI, Björkander J, Oldfors A. Inclusion body myositis: clinical, morphological, physiological and laboratory findings in 18 cases. Acta Neurol Scand. 1994; 89(2):123-131. [PubMed: 8191875]

8. Sayers ME, Chou SM, Calabrese LH. Inclusion body myositis: analysis of 32 cases. J Rheumatol. 1992; 19(9):1385-1389. [PubMed: 1331441]

9. Amato AA, Gronseth GS, Jackson CE, et al. Inclusion body myositis: clinical and pathological boundaries. Ann Neurol. 1996; 40(4):581-586. [PubMed: 8871577]

10. Barohn RJ, Amato AA. Inclusion body myositis. Curr Treat Options Neurol. 2000; 2(1):7-12. [PubMed: 11096732]

11. Needham M, James I, Corbett A, et al. Sporadic inclusion body myositis: phenotypic variability and influence of HLA-DR3 in a cohort of 57 Australian cases. J Neurol Neurosurg Psychiatry. 2008; 79(9):1056-1060. [PubMed: 18258695] 
12. Larue S, Maisonobe T, Benveniste O, et al. Distal muscle involvement in granulomatous myositis can mimic inclusion body myositis. J Neurol Neurosurg Psychiatry. 2011; 82(6):674-677. [PubMed: 20562458]

13. Barohn RJ, Amato AA, Sahenk Z, Kissel JT, Mendell JR. Inclusion body myositis: explanation for poor response to immunosuppressive therapy. Neurology. 1995; 45(7):1302-1304. [PubMed: 7617187]

14. Hilton-Jones D, Miller A, Parton M, Holton J, Sewry C, Hanna MG. Inclusion body myositis: MRC Centre for Neuromuscular Diseases, IBM workshop, London, 13 June 2008. Neuromuscul Disord. 2010; 20(2):142-147. [PubMed: 20074951]

15. Griggs RC, Askanas V, DiMauro S, et al. Inclusion body myositis and myopathies. Ann Neurol. 1995; 38(5):705-713. [PubMed: 7486861]

16. Amato AA, Barohn RJ. Idiopathic inflammatory myopathies. Neurol Clin. 1997; 15(3):615-648. [PubMed: 9227956]

17. Garlepp MJ, Laing B, Zilko PJ, Ollier W, Mastaglia FL. HLA associations with inclusion body myositis. Clin Exp Immunol. 1994; 98(1):40-45. [PubMed: 7923882]

18. Degardin A, Morillon D, Lacour A, Cotten A, Vermersch P, Stojkovic T. Morphologic imaging in muscular dystrophies and inflammatory myopathies. Skeletal Radiol. 2010; 39(12):1219-1227. [PubMed: 20449587]

19. Cox FM, Reijnierse M, van Rijswijk CS, Wintzen AR, Verschuuren JJ, Badrising UA. Magnetic resonance imaging of skeletal muscles in sporadic inclusion body myositis. Rheumatology (Oxford). 2011; 50(6):1153-1161. [PubMed: 21288962]

20. Maetzler W, Reimold M, Schittenhelm J, et al. Increased [11C]PIB-PET levels in inclusion body myositis are indicative of amyloid beta deposition. J Neurol Neurosurg Psychiatry. 2011; 82(9): 1060-1062. [PubMed: 20732867]

21. van der Meulen MF, Hoogendijk JE, Moons KG, Veldman H, Badrising UA, Wokke JH. Rimmed vacuoles and the added value of SMI-31 staining in diagnosing sporadic inclusion body myositis. Neuromuscul Disord. 2001; 11(5):447-451. [PubMed: 11404115]

22. Greenberg SA, Pinkus GS, Amato AA, Pinkus JL. Myeloid dendritic cells in inclusion-body myositis and polymyositis. Muscle Nerve. 2007; 35(1):17-23. [PubMed: 16969836]

23. Greenberg SA. Inclusion body myositis. Curr Opin Rheumatol. 2011; 23(6):574-578. [PubMed: 21885973]

24. Greenberg SA, Sanoudou D, Haslett JN, et al. Molecular profiles of inflammatory myopathies. Neurology. 2002; 59(8):1170-1182. [PubMed: 12391344]

25. Greenberg SA, Bradshaw EM, Pinkus JL, et al. Plasma cells in muscle in inclusion body myositis and polymyositis. Neurology. 2005; 65(11):1782-1787. [PubMed: 16344523]

26. Salajegheh M, Lam T, Greenberg SA. Autoantibodies against a $43 \mathrm{KDa}$ muscle protein in inclusion body myositis. PLoS ONE. 2011; 6(5):e20266. [PubMed: 21629782]

27. Askanas V, Engel WK. Inclusion-body myositis: amyodegenerative conformational disorder associated with Abeta, protein misfolding, and proteasome inhibition. Neurology. 2006; 66 Suppl 1(2):S39-S48. [PubMed: 16432144]

28. Askanas V, Engel WK. Sporadic inclusion-body myositis: conformational multifactorial ageingrelated degenerative muscle disease associated with proteasomal and lysosomal inhibition, endoplasmic reticulum stress, and accumulation of amyloid- $\beta 42$ oligomers and phosphorylated tau. Presse Med. 2011; 40(4 Pt 2):e219-e235. [PubMed: 21392932]

29. Salajegheh M, Pinkus JL, Nazareno R, Amato AA, Parker KC, Greenberg SA. Nature of "Tau" immunoreactivity in normal myonuclei and inclusion body myositis. Muscle Nerve. 2009; 40(4): 520-528. [PubMed: 19626672]

30. Nogalska A, D'Agostino C, Engel WK, Klein WL, Askanas V. Novel demonstration of amyloid- $\beta$ oligomers in sporadic inclusion-body myositis muscle fibers. Acta Neuropathol. 2010; 120(5): 661-666. [PubMed: 20711838]

31. Amato AA, Barohn RJ. Inclusion body myositis: old and new concepts. J Neurol Neurosurg Psychiatry. 2009; 80(11):1186-1193. [PubMed: 19864656]

32. Salajegheh M, Pinkus JL, Taylor JP, et al. Sarcoplasmic redistribution of nuclear TDP-43 in inclusion body myositis. Muscle Nerve. 2009; 40(1):19-31. [PubMed: 19533646] 
33. Verma A, Bradley WG, Ringel SP. Treatment-responsive polymyositis transforming into inclusion body myositis. Neurology. 2008; P060:19.

34. Benveniste $\mathrm{O}$, Guiguet $\mathrm{M}$, Freebody J, et al. Long-term observational study of sporadic inclusion body myositis. Brain. 2011; 134(Pt 11):3176-3184. [PubMed: 21994327]

35. Amato AA, Barohn RJ, Jackson CE, Pappert EJ, Sahenk Z, Kissel JT. Inclusion body myositis: treatment with intravenous immunoglobulin. Neurology. 1994; 44(8):1516-1518. [PubMed: 8058161]

36. Dalakas MC, Sonies B, Dambrosia J, Sekul E, Cupler E, Sivakumar K. Treatment of inclusionbody myositis with IVIg: a double-blind, placebo-controlled study. Neurology. 1997; 48(3):712716. [PubMed: 9065553]

37. Dalakas MC, Koffman B, Fujii M, Spector S, Sivakumar K, Cupler E. A controlled study of intravenous immunoglobulin combined with prednisone in the treatment of IBM. Neurology. 2001; 56(3):323-327. [PubMed: 11171896]

38. Muscle Study Group. Randomized pilot trial of betaINF1a (Avonex) in patients with inclusion body myositis. Neurol (Tokyo). 2001; 57:1566-1570.

39. Muscle Study Group. Randomized pilot trial of high-dose betaINF-1a in patients with inclusion body myositis. Neurol (Tokyo). 2004; 63:718-720.

40. Badrising UA, Maat-Schieman ML, Ferrari MD, et al. Comparison of weakness progression in inclusion body myositis during treatment with methotrexate or placebo. Ann Neurol. 2002; 51(3): 369-372. [PubMed: 11891832]

41. Lindberg C, Trysberg E, Tarkowski A, Oldfors A. Anti-T-lymphocyte globulin treatment in inclusion body myositis: a randomized pilot study. Neurology. 2003; 61(2):260-262. [PubMed: 12874415]

42. Rutkove SB, Parker RA, Nardin RA, Connolly CE, Felice KJ, Raynor EM. A pilot randomized trial of oxandrolone in inclusion body myositis. Neurology. 2002; 58(7):1081-1087. [PubMed: 11940697]

43. Barohn RJ, Herbelin L, Kissel JT, et al. Pilot trial of etanercept in the treatment of inclusion-body myositis. Neurology. 2006; 66 Suppl 1(2):S123-S124. [PubMed: 16432140]

44. Dalakas MC, Rakocevic G, Schmidt J, et al. Effect of Alemtuzumab (CAMPATH 1-H) in patients with inclusion-body myositis. Brain. 2009; 132(Pt 6):1536-1544. [PubMed: 19454532]

45. Sancricca C, Mora M, Ricci E, Tonali PA, Mantegazza R, Mirabella M. Pilot trial of simvastatin in the treatment of sporadic inclusion-body myositis. Neurol Sci. 2011; 32(5):841-847. [PubMed: 21695654]

46. Cox FM, Titulaer MJ, Sont JK, Wintzen AR, Verschuuren JJ, Badrising UA. A 12-year follow-up in sporadic inclusion body myositis: an end stage with major disabilities. Brain. 2011; 134(Pt 11): 3167-3175. [PubMed: 21908393]

47. Sekul EA, Dalakas MC. Inclusion body myositis: new concepts. Semin Neurol. 1993; 13(3):256263. [PubMed: 8272596]

48. Arnardottir S, Alexanderson H, Lundberg IE, Borg K. Sporadic inclusion body myositis: pilot study on the effects of a home exercise program on muscle function, histopathology and inflammatory reaction. J Rehabil Med. 2003; 35(1):31-35. [PubMed: 12610846]

49. Johnson GL, et al. The effectiveness of an individualized, home-based functional exercise program for patients with sporadic inclusion body myositis. J Clin Neuromuscul Dis. 2007; 8:187-194.

50. Johnson LG, Collier KE, Edwards DJ, et al. Improvement in aerobic capacity after an exercise program in sporadic inclusion body myositis. J Clin Neuromuscul Dis. 2009; 10(4):178-184. [PubMed: 19494728]

51. Takarada Y, Takazawa H, Sato Y, Takebayashi S, Tanaka Y, Ishii N. Effects of resistance exercise combined with moderate vascular occlusion on muscular function in humans. J Appl Physiol. 2000; 88(6):2097-2106. [PubMed: 10846023]

52. Wernbom M, Augustsson J, Raastad T. Ischemic strength training: a low-load alternative to heavy resistance exercise? Scand J Med Sci Sports. 2008; 18(4):401-416. [PubMed: 18466185]

53. Gualano B, Neves M Jr, Lima FR, et al. Resistance training with vascular occlusion in inclusion body myositis: a case study. Med Sci Sports Exerc. 2010; 42(2):250-254. [PubMed: 19927034] 

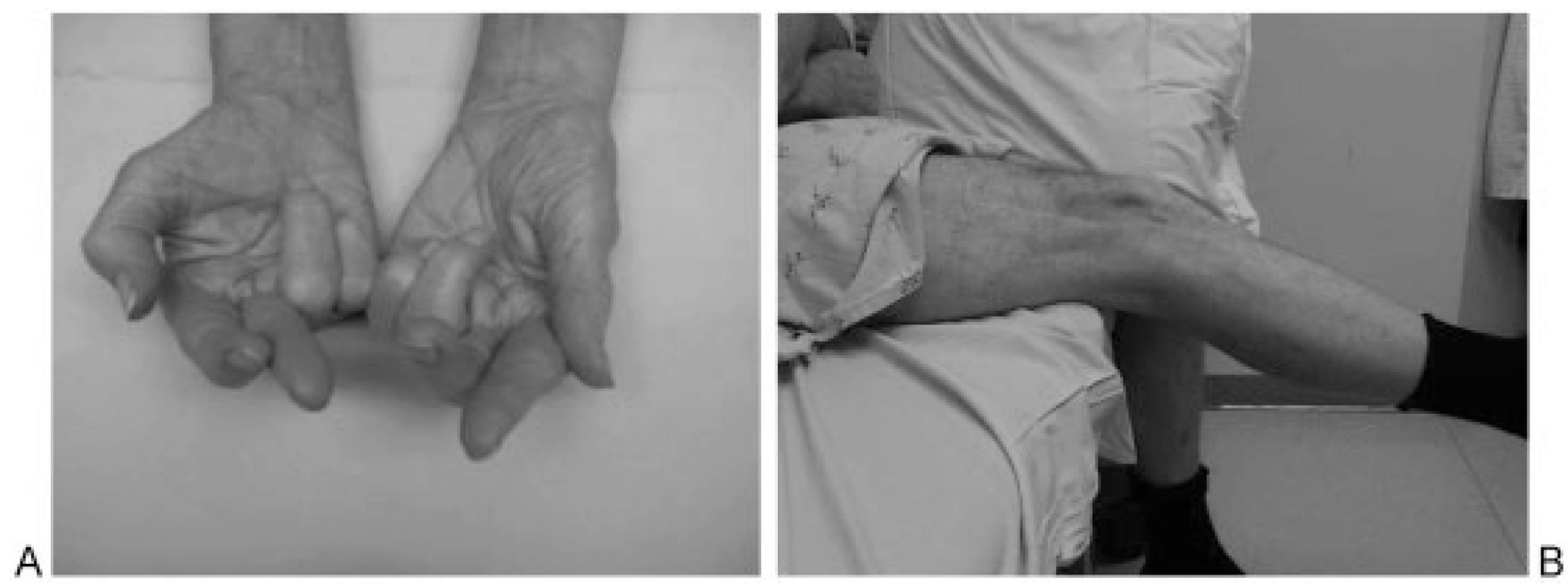

Figure 1.

(A) Asymmetric finger flexor weakness in inclusion body myositis (IBM). (B) Quadriceps muscle atrophy with inability to fully extend the knee in IBM. 

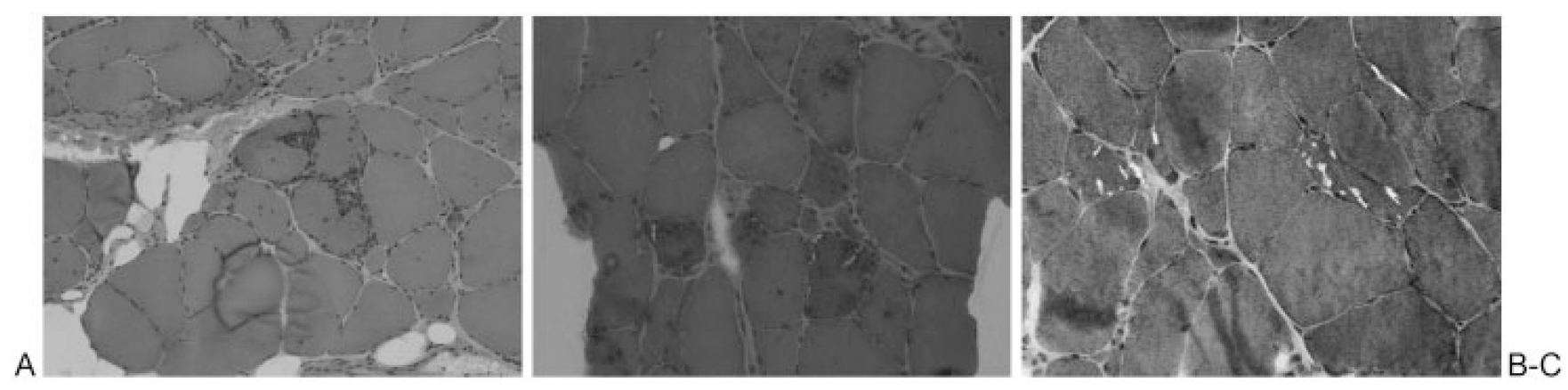

Figure 2.

(A) Polymyositis: Inflammatory infiltrates invading nonnecrotic fibers (hematoxylin \& eosin). (B) Multiple vacuolated fibers in inclusion body myositis (IBM) (hematoxylin \& eosin). (C) IBM muscle fibers with multiple rimmed vacuoles (modified Gomori trichrome). 


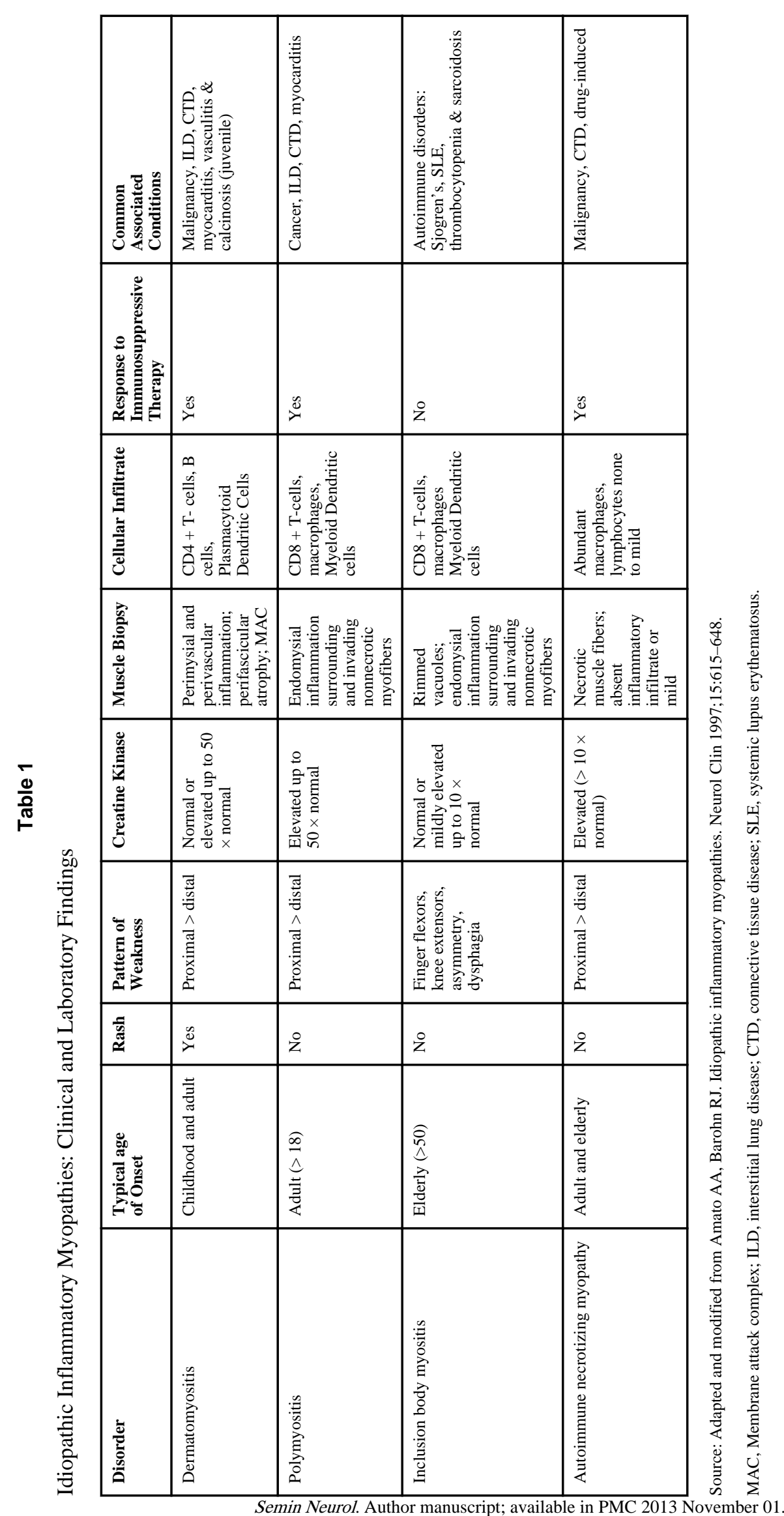


Table 2

\section{Griggs Diagnostic Criteria for Inclusion Body Myositis}

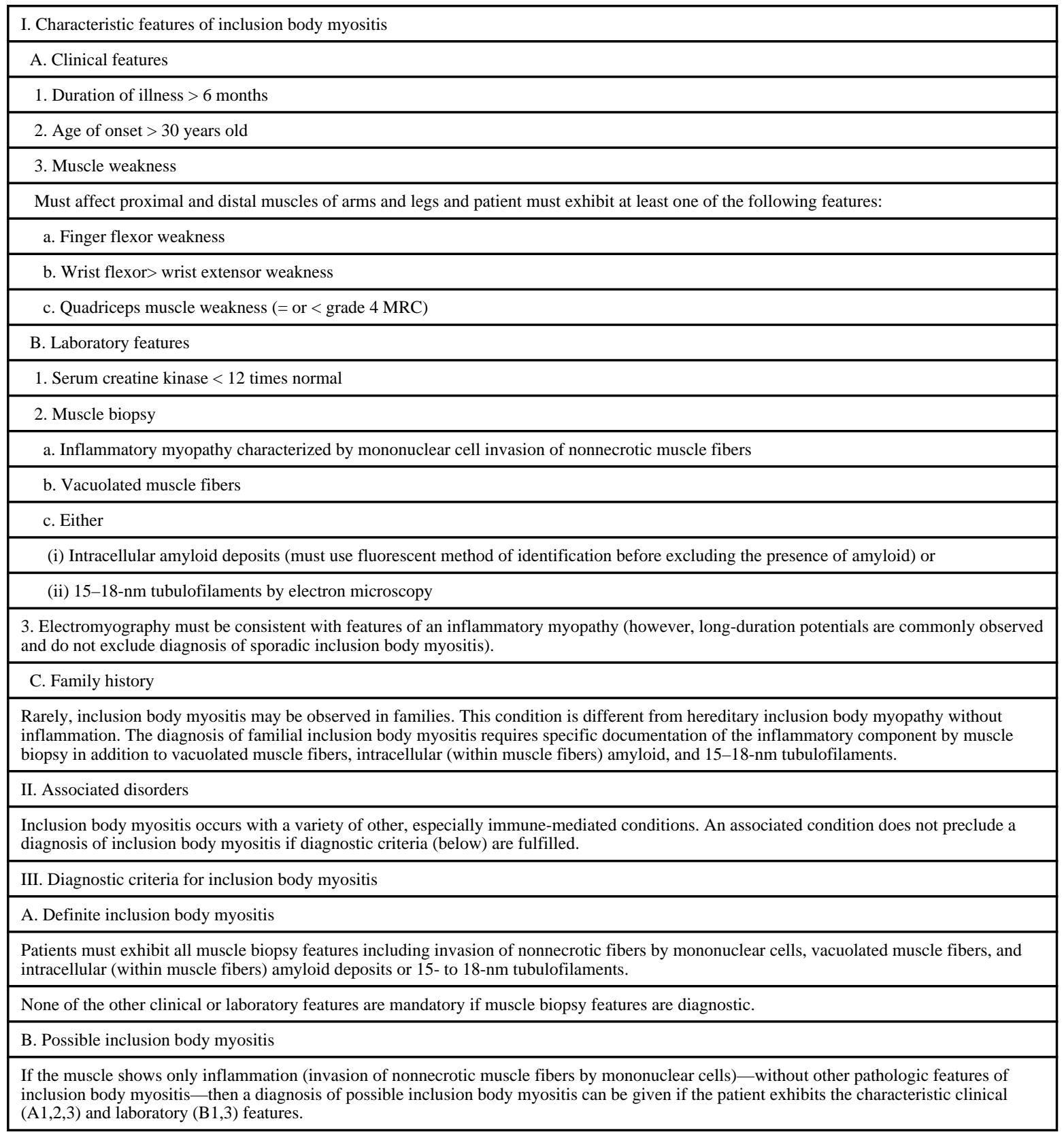

Source: Adapted and modified from Griggs RC, Askanas V, DiMauro S, Engel A, Karpati G, Mendell JR, Rowland LP. Inclusion body myositis andmyopathies. Ann Neurol 1995;38(5):705-713. 
Table 3

\section{Modified Inclusion Body Myositis Diagnostic Criteria 2008}

\begin{tabular}{|l|}
\hline Pathologically defined inclusion body myositis \\
\hline $\begin{array}{l}\text { C Conforming to the Griggs criteria }{ }^{15} \text { : Invasion of nonnecrotic fibers by mononuclear cells and rimmed vacuoles, and either intracellular } \\
\text { amyloid deposits or } 15-\text { to } 18 \text {-nm filaments. }\end{array}$ \\
\hline Clinically defined inclusion body myositis \\
\hline • Clinical features \\
\hline O Duration of weakness $>12$ months \\
\hline O Age > 35 years \\
\hline O Weakness of finger flexion > shoulder abduction AND of knee extension > hip flexion \\
\hline • Pathologic features \\
\hline O Invasion of nonnecrotic fibers by mononuclear cells or rimmed vacuoles or increased \\
\hline O MHC-1, but no intracellular amyloid deposits or 15 - to 18 -nm filaments \\
\hline Possible IBM \\
\hline • Clinical criteria \\
\hline O Duration of weakness $>12$ months \\
\hline O Age > 35 years \\
\hline O Weakness of finger flexion > shoulder abduction OR of knee extension > hip flexion \\
\hline • Pathologic criteria \\
\hline O Invasion of nonnecrotic fibers by mononuclear cells or rimmed vacuoles or increased \\
\hline O MHC-1, but no intracellular amyloid deposits or 15 - to 18 -nm filaments \\
\hline
\end{tabular}

Source: Adapted and modified from Hilton-Jones D, Miller A, Parton M, et al. Inclusion body myositis. Neuromuscul Disord 2010;20(2):142-147. 